\title{
Erratum: Dissecting reaction calculations using halo effective field theory and $a b$ initio input [Phys. Rev. C 98, 034610 (2018)]
}

\author{
P. Capelø, D. R. Phillips $\odot$, and H.-W. Hammer®
}

(Received 1 December 2021; published 25 January 2022)

DOI: 10.1103/PhysRevC.105.019901

In the original paper, we have presented a new analysis of the breakup of one-neutron halo nuclei by coupling a model of the projectile motivated by halo effective field theory with a fully dynamical treatment of the reaction using the dynamical eikonal approximation. The agreement with data at intermediate energy on the archetypical halo nucleus ${ }^{11} \mathrm{Be}$ is excellent. It is independent of the cutoff in the effective ${ }^{10} \mathrm{Be}-n$ interaction used to describe the halo structure. The extension of this paper to other reactions [1,2] has shown there is a typographical error in Table I of the original paper: for the cutoff $\sigma=2 \mathrm{fm}$, the depth of the surface term of the next-to-leading order ${ }^{10} \mathrm{Be}-n$ interaction should read $V_{s 1 / 2}^{(2)}=+3 \mathrm{MeV} \mathrm{fm}^{-2}$. The units for that term in Tables I, II, IV, V, and VI should read $\mathrm{MeV} \mathrm{fm}^{-2}$. None of these changes affect the results presented in the paper.

We thank C. Hebborn and I. Alnamlah for bringing these issues to our attention and checking our results.

[1] L. Moschini and P. Capel, Phys. Lett. B 790, 367 (2019).

[2] C. Hebborn and P. Capel, Phys. Rev. C 104, 024616 (2021). 